\title{
Ionic-Liquid Matrices for Quantitative Analysis by MALDI-TOF Mass Spectrometry
}

\author{
Ying L. Li and Michael L. Gross \\ Department of Chemistry, Washington University, St. Louis, Missouri, USA
}

Ionic liquid matrices (ILMs) were tested as MALDI matrices for quantification of oligodeoxynucleotides (ODNs), peptides, and small proteins. Good calibrations with high linearity and reproducibility were achieved over a broad concentration range for all the tested ILMs in spite of their different physical states. However, the standard deviation is higher for ILMs that are solid with visible crystals. The experimental results indicate various ILMs have different sensitivity owing to changes in their cation components. More importantly, we found that the slopes of the calibration curves correlate with the inverse of the peptide molecular weights, presenting an opportunity to predict a priori, the relative sensitivities (slopes of calibration plots) for various analytes that have similar hydrophobicites. (J Am Soc Mass Spectrom 2004, 15, 1833-1837) () 2004 American Society for Mass Spectrometry

$\mathrm{R}$ oom-temperature ionic liquids (RTILS) are salts with melting points at or below room temperature. Typically, an RTIL consists of a nitrogen or phosphorus-containing organic cation and a large organic or inorganic anion [1]. They have been used as novel solvent systems in organic chemistry, for liquidliquid extraction, as a solvent in electrochemistry, and as a new class of stationary phases for gas chromatography $[2,3]$.

Our laboratory, in collaboration with that of Armstrong, introduced a class of specially designed RTILs that are capable of absorbing laser light and transferring protons to the analyte as matrices for MALDI mass spectrometry [4]. We define them as class II RTILs to differentiate them from class I RTILs (i.e., those that are not suitable as MALDI matrices). Class II RTILs have as the anion the conjugate base of classical solid matrices and, therefore, are chromophores. They also have higher hydrogen bond acidity [5]. We now name the class II RTILs as "ionic liquid matrices" (ILMs) to distinguish them from other classes of RTILs that are not suitable for MALDI.

With Armstrong [4], we reported the synthesis of 20 different ILMs as nonvolatile amine salts of $\alpha$-cyano-4hydroxycinnamic acids (CHCA) and sinapinic acids (SA). These ILMs proved to be useful for the analysis of peptides, proteins and synthetic polymers. Their success derives from their high solubilizing power, low vapor pressure, broad liquid range (remain liquid to $200{ }^{\circ} \mathrm{C}$ and higher), good spot homogeneity, and con-

Published online November 2, 2004

Address reprint requests to Dr. M. L. Gross, Department of Chemistry, Washington University, One Brookings Drive, St. Louis, MO 63130, USA. E-mail: mgross@wustl.edu comitant shot-to-shot reproducibility compared to classical solid matrices. Their properties minimize searching for a "sweet spot."

Armstrong and coworkers [6] extended the use of ILMs by documenting the advantages of organic salts of 3-hydroxypicolinic acids (3-HPA) and 2,5-dihydroxy benzoic acids (2,5-DHB) as matrices for oligodeoxynucleotides (ODNs). Stahl and coworkers [7] more recently conducted a thorough evaluation of the ILMs butylammonium 2,5-dihydroxybenzoate (DHBB), butylammonium $\alpha$-cyano-4-hydroxycinnamate (CHCAB), and triethylammonium sinapinate (SinTri). They demonstrated improved reproducibility from 90 different measurements for sample preparations involving DHBB and 2,5-DHB, CHCAB and CHCA. They also found that the ILM, DHBB, has the same broad applicability as solid DHB for oligosaccharides, glycoconjugates, peptides, proteins, and synthetic polymers.

Other advantages of ILMs are lower MALDIinduced fragmentation and their ability to serve as both the reaction cosolvent and the MALDI matrix for conducting submicroliter enzymatic reactions [7]. The sensitivity of MALDI with ILMs, however, varies on a case-by-case basis. Armstrong et al. [4] reported certain ILMs (i.e., anilinium $\alpha$-cyano-4-hydroxycinnamate, $N, N$-diethyl benzenaminium $\alpha$-cyano-4-hydroxycinnamate) outperformed solid matrices and gave better detection limits for bradykinin. Gross and Li [8] reported improved detection limit (one order of magnitude) for phospholipids by using certain kinds of ILMs (e.g., anilinium $\alpha$-cyano-4-hydroxycinnamate); while Stahl and coworkers [7] demonstrated that low to mid picomole detection limits can be achieved for peptides and oligosaccharides when using the ILMs, CHCAB and DHBB. This detection 
limit is 10 to 10000 -fold worse than those achieved with their solid analogs. The detection limit appears to depend both on the nature of the analyte and the ILM because certain ILMs interact differently with various analytes. Nevertheless, even for the ILMs that gave poorer detection limits, the problem can be partly compensated by using "in-matrix" preconcentration or a smaller matrix droplet [9] (e.g., $\mu$ m-sized sample spots [10]).

An important issue for MALDI is the ability to quantify over a wide range of concentration with predictable response for various analytes. Poor shot-to-shot reproducibility, stemming from variability in the distribution and surface of the matrix [11], limits the application of solid-matrix MALDI in quantification. Nevertheless, with carefully designed sample preparation methods (e.g., employing a thin-layer matrix [12] or using a matrix/comatrix to increase sample spot homogeneity [13]), some groups have achieved satisfactory quantitative results for ODNs over a dynamic range of at least one order of magnitude. Other sample preparations aimed at providing more homogeneous crystallization between analyte and matrix include "seed-layer" [14-16], two-layer (underlayer/overlayer) [17, 18], fast evaporation of matrix solvent to produce a thin-layer matrix film [19], and electrospray deposition of the matrix/sample [20]. Although improvements in quantification were achieved $[12,13]$, these sample preparation methods were strongly dependent on the nature of the sample and the expertise of the spectrometrist. Further improvements are clearly needed.

One obvious means of improving homogeneity is to use liquid matrices (e.g., glycerol) but unfortunately they do not have strong absorbance in the UV, making them not useful for commonly practiced UV MALDI. It is possible to circumvent this problem by "doping" the transparent matrix with materials that have strong UV absorbance; example dopants are ultrafine cobalt powder [21], suspended carbon particles [22], or solid matrices that are combined with a solubilizing reagent to improve miscibility [9]. ILMs are another example of the strategy to use liquid matrices. The first attempt to evaluate quantification with ILMs was by Tholey and coworkers [23], who reported quantification of a lowmolecular-weight compound (i.e., glutamine) by using an isotopically labeled internal standard. They obtained improved calibration, lower standard deviations, lower number of values to be rejected as outliers, and shortened measurement time compared to the use of the corresponding solid matrix. Our focus with the ILMs is to extend the quantification to a wider range of analyte types, including substances important in biology.

\section{Experimental}

\section{Materials}

$\alpha$-Cyano-4-hydroxycinnamic acid (CHCA), 1-methylimidazole, aniline, pyridine, $N, N$-diethylamine, tri- ethylamine, tripropylamine, tributylamine, and trifluoroacetic acid (TFA) were purchased from Aldrich (Milwaukee, WI). Bradykinin, Tyr-bradykinin, substance $\mathrm{P}$, melittin, and bovine insulin were obtained from Sigma (St. Louis, MO). Oligonucleotides 5'$\mathrm{d}$ (CTTTCCTC) and 5'-d(TCTTCCCTT) were synthesized by Integrated DNA Technologies (Coralville, IA). HPLC grade organic solvents were obtained from Fisher (St. Louis, MO). All chemicals were of the highest available purity grade and were used without further purification.

RTILs of CHCA were prepared as previously reported [4].

\section{Sample Preparation for MALDI-TOF MS Analysis}

All ionic liquids were dissolved in a mixture of acetonitrile/water (2:1, vol/vol, $0.1 \%$ TFA) at a concentration of $0.5 \mathrm{M}$. If the required amount exceeded the solubility of the RTIL, a saturated solution was used. Oligonucleotides 5'-d(CTTTCCTC) (MW = 2310.4), bradykinin (MW = 1059.6), melittin ( $\mathrm{MW}=2844.8)$, and bovine insulin ( $\mathrm{MW}=5729.6)$ were chosen as test analytes while 5'-d(TCTTCCCTT) (MW = 2614.5), Tyr-bradykinin $(\mathrm{MW}=1222.6)$, and substance $\mathrm{P}(\mathrm{MW}=1346.7)$ were chosen to be the internal standards. They were dissolved in deionized water and diluted to 40; 80; 120; $160 ; 200 ; 400 \mu \mathrm{M}$ from a $1000-\mu \mathrm{M}$ stock solution; the $40-\mu \mathrm{M}$ solution was further diluted to $4 ; 8 ; 16 ; 24 ; 32 \mu \mathrm{M}$ solutions. The internal standards $\left(5^{\prime}-\mathrm{d}(\mathrm{TCTTCCCTT})\right.$, Tyr-bradykinin, and substance $\mathrm{P}$ ) were kept at a concentration of $40 \mu \mathrm{M}$. A $10-\mu \mathrm{L}$ aliquot of the analyte and internal standard mixture solution was mixed with the same volume of the ILM solution to give a final spotting solution for which the final concentration of the analyte was in the range of 1.0 to $100 \mu \mathrm{M}$.

For each solution of analyte/internal standard, three sample spots were prepared by loading $1 \mu \mathrm{L}$ of the sample/matrix solution on the MALDI plate. For one spot, 100 laser shots were taken from a randomly chosen position, and this procedure was repeated for the other two sample spots. All spectra were smoothed (by using "noise filter") and base line corrected with the Data Explorer software. The peak-height ratios of the analyte relative to those of the internal standard were calculated and averaged from the three measurements at each concentration.

\section{Instrumentation}

A Voyager DE-RP mass spectrometer (PerSeptive Biosystems, Framingham, MA) equipped with a nitrogen laser (337-nm, 3-ns pulse) was operated in the linear positive-ion mode. The irradiance of the laser was adjusted to achieve optimum signal-to-noise ratio and minimum MALDI-induced fragmentation of analyte molecules. The acceleration voltage was $20 \mathrm{kV}$, grid voltage was $95 \%$, guide-wire voltage was $0.1 \%$ of the accelerating voltage, and the delay time was 200 ns. 


\section{Results and Discussion \\ Oligodeoxynucleotides (ODNs)}

We chose ODNs as our first test analytes to evaluate quantification with ILMs compared with a multicomponent matrix system that was previously used [13]. An appropriate internal standard for MALDI quantification should possess physical and chemical properties that are common with those of the analytes [24, 25]. Previous research indicates that an ODN that has a similar sequence to that of the analyte is a good internal standard when solid-state matrices are used $[12,13,26]$. We chose $5^{\prime}-\mathrm{d}$ (TCTTCCCTT) as the internal standard to quantify 5 '-d(CTTTCCTC) and evaluated the ILMs, 1-methyl imidazolium $\alpha$-cyano-4-hydroxy cinnamate (1-MeIm-CHCA), which is liquid at room temperature, diethyl ammonium $\alpha$-cyano-4-hydroxy cinnamate (DieCHCA), and pyridinium $\alpha$-cyano-4-hydroxy cinnamate (Py-CHCA), which are solids at room temperature. (For more information about the physical states of ILMs, refer to Table 2 in Reference [4]). 1-MeIm-CHCA, which stayed liquid at room temperature, exhibited glycerollike viscosity. Because the original form is viscous, we dissolved it in acetonitrile/water (2:1, vol/vol, $0.1 \%$ TFA) at a concentration of $0.5 \mathrm{M}$ to improve the minimum volume $(\sim 1 \mu \mathrm{L})$ that could be practically handled. The concentration of $5^{\prime}-\mathrm{d}$ (TCTTCCCTT) was constant $(10 \mu \mathrm{M})$, whereas that of $5^{\prime}-\mathrm{d}($ CTTTCCTC) was varied over the range of $25(2 \mu \mathrm{M}$ to $50 \mu \mathrm{M})$. The mass resolving power slightly decreased when ILMs were used instead of solid matrices, which is consistent with an observation by Stahl et al. [7]. We simply measured the signal intensities rather than the integrals (peak areas), as was suggested for quantitative MALDI work [27]. Plots of the ratios of peak height for test analyte and standard versus the amount of analyte loaded on the sample plate show a good linear correlation. The resulting calibration curves are linear with correlation coefficients $\left(\mathrm{R}^{2}\right)$ of 0.994 to 0.996 (Figure 1). The relative

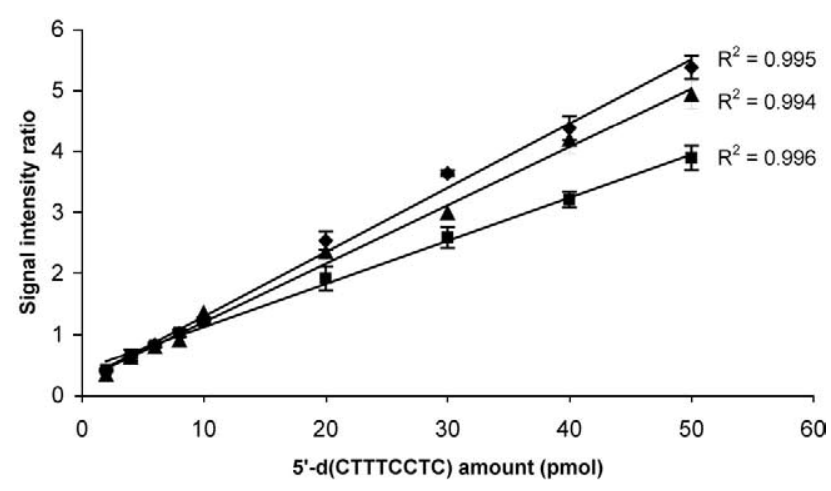

Figure 1. Calibration curves for the MALDI quantification of 5'-d(CTTTCCTC) using ILMs: 1-MeIm-CHCA (filled diamond) $(\mathrm{m}=0.11, \mathrm{~b}=0.24)$, Die-CHCA (filled triangle) $(\mathrm{m}=0.10, \mathrm{~b}=$ $0.25)$, and Py-CHCA (filled square) $(\mathrm{m}=0.07, \mathrm{~b}=0.42)(\mathrm{m}$ : slope of calibration curve, b: intercept of calibration curve). The $5^{\prime}-$ $\mathrm{d}$ (TCTTCCCTT) amount was fixed at $10 \mathrm{pmol}$.

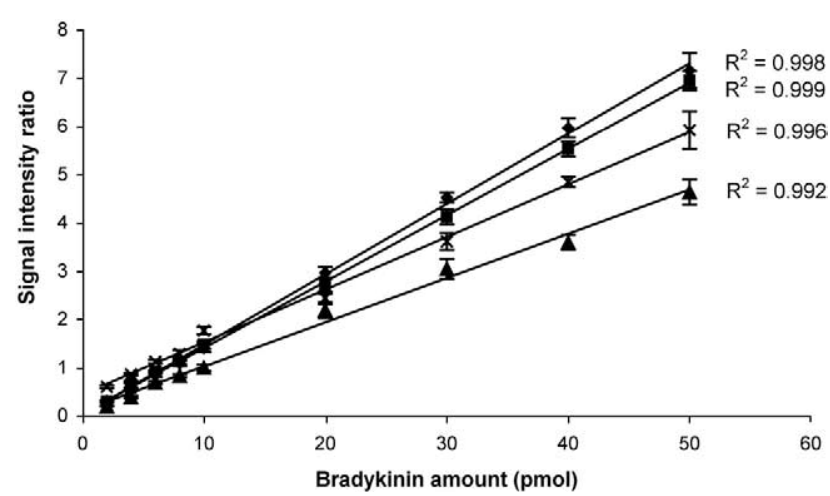

Figure 2. Calibration curves for the MALDI quantification of bradykinin using ILMs: Trie-CHCA (filled diamond) $(\mathrm{m}=0.15$, $\mathrm{b}=0.02)$, Trip-CHCA (filled square) $(\mathrm{m}=0.14, \mathrm{~b}=0.04)$, Trib-CHCA $(x)(m=0.11, b=0.44)$, and Diea-CHCA (filled triangle) $(\mathrm{m}=0.09, \mathrm{~b}=0.11)(\mathrm{m}$ : slope of calibration curve; $\mathrm{b}$ : intercept of calibration curve). The Tyr-bradykinin amount was fixed at 10 pmol.

standard deviations (RSD) for quantification range from 1 to $6 \%$ for $1-\mathrm{MeIm}-\mathrm{CHCA}, 1$ to $8 \%$ for Die-CHCA, and 2 to $15 \%$ for Py-CHCA.

These results indicate that ILMs offer good reproducibility over a large dynamic range for ODN quantification. The higher RSDs for Py-CHCA are probably because its physical state is solid at room temperature and less homogeneous (we did observe crystals on the sample spot). This phenomenon agrees with what was previously reported [4, 6]: many ILMs are solid at room temperature with visible crystals. Die-CHCA, which is also solid at room temperature, produced an extremely thin and flat sample spot in contrast to that produced with Py-CHCA. The matrix crystals were much smaller and more uniformly distributed. The homogeneity of Die-CHCA, as a low-melting-point solid, exceeded that of highly crystallized solid matrices [4], explaining why it performed similarly to liquid matrices. Furthermore, different slopes (0.07-0.11) and intercepts (0.24-0.42) indicate that different ILMs have a sensitivity that depends on the nature of the cation constituent (imidazole, pyridine rings, and alkyl chains).

\section{Peptides and Proteins}

To test the applicability of these matrices to other classes of biomolecules, we quantified the polypeptide bradykinin by using various ILMs. Tholey and coworkers [23], in the analysis of single amino acid, required an isotopically labeled analog of the analyte as the internal standard to achieve good calibration. Unfortunately, isotopically labeled standards, although reasonably applicable to small molecules, are more difficult to obtain for larger biomolecules. To work within this constraint, we chose Tyr-bradykinin, which differs from bradykinin by one amino acid, to quantify bradykinin. We tested the ILMs, triethyl ammonium $\alpha$-cyano-4-hydroxy cinnamate (Trie-CHCA), tripropyl ammonium $\alpha$-cyano4-hydroxy cinnamate (Trip-CHCA), tributyl ammo- 


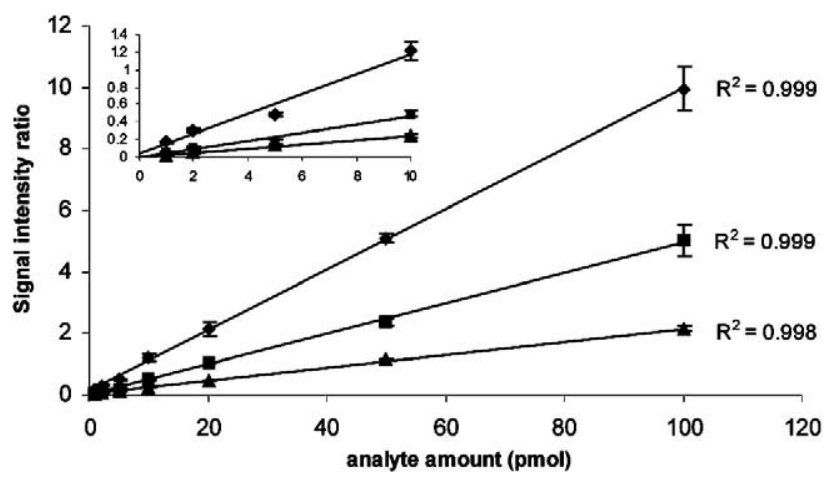

Figure 3. Calibration curves for the MALDI quantification of bradykinin (filled diamond) $(\mathrm{m}=0.10, \mathrm{~b}=0.12)$, melittin (filled square) $(\mathrm{m}=0.05, \mathrm{~b}=-0.02)$, and bovine insulin (filled triangle) $(\mathrm{m}=0.02, \mathrm{~b}=0.02)(\mathrm{m}$ : slope of calibration curve; $\mathrm{b}$ : intercept of calibration curve) by using ILM Die-CHCA as MALDI matrix. The amount of analyte ranged from $1 \mathrm{pmol}$ to $100 \mathrm{pmol}$. The substance $\mathrm{P}$ amount was fixed at $10 \mathrm{pmol}$. The inset magnifies the calibration at low amounts.

nium $\alpha$-cyano-4-hydroxy cinnamate (Trib-CHCA), and diethyl anilinium $\alpha$-cyano-4-hydroxy cinnamate (DieaCHCA), which are all liquids and exhibit glycerol-like viscosity at room temperature. The calibration curves are linear with correlation coefficients $\left(\mathrm{R}^{2}\right)$ of 0.992 to 0.998 (Figure 2). The relative standard deviations (RSDs) for quantification are 2 to $6 \%$ for Trie-CHCA, 1 to $6 \%$ for Trip-CHCA, 3 to $7 \%$ for Trib-CHCA, and 2 to $7 \%$ for Diea-CHCA. These results indicate that ILMs can achieve high reproducibility and sensitivity for polypeptide quantification without an isotopically labeled internal standard.

The use of different ILMs affords different slopes $(0.09-0.15)$ and intercepts $(0.02-0.44)$, indicating that the use of various ILMs leads to different sensitivities owing to changes in the structures of ILMs' cation components. However, since all the ILMs we tested give satisfactory linear relationships and standard deviations for the calibration curves despite differences in cation constituent, the success of the ILMs does not depend strongly on the cation choice.

Can one use an internal standard with structures and molecular weights that are different from those of the analytes? Others showed that when internal standards with properties different than those of the analyte are used, the accuracy and linearity of the calibration suffers $[26,28]$. When the parameters of the system are controlled (primarily the laser irradiance), however, the internal standard can differ from the analyte [29]. In the next test of ILMs, we used the same internal standard, substance $P$, to quantify peptides that have considerably different structures but are similar in their hydrophobicities. The test peptides are bradykinin, melittin, and bovine insulin, with molecular weights of approximately 1000, 3000, and 6000 $\mathrm{Da}$, respectively. Their hydrophobicities, however, are similar (i.e., their Bull and Breese values [30] are -163 , -104 , and $-172 \mathrm{cal} / \mathrm{mol}$, respectively) to each other and to that of the internal standard (i.e., $-130 \mathrm{cal} / \mathrm{mol}$ ).

Although all of the ILMs allowed the test analytes to dissolve readily, they varied significantly in their ability to promote ionization of various analytes [4]. We found that a subtle difference in the cation component sometimes leads to significant changes in MS response, but the reason for this is not clear and should be the subject of further investigation. We chose ILM Die-CHCA as the matrix for this application because its use gives the best signal for the analytes and internal standard. Although its physical state at room temperature is solid, as previously discussed, it produces very fine and homogeneous crystals over the entire sample spot, very much as a liquid would. The calibration curves that result from its use have good linearity and reproducibility [i.e., correlation coefficients $\left(R^{2}\right)$ are 0.99$]$ over a concentration range of a factor of 100 (Figure 3). The standard deviations (SDs) for peak-height ratios are small (Table 1). All the SD values except the first and last points, which are known to be prone to large errors because they represent the ratio extremes [27, 28], have good reproducibility. We did a similar calibration by using CHCA as the matrix (data not shown), but the plots are not linear and show saturation at modest concentration ratios, and give RSD values as high as $50 \%$.

Perhaps of more importance for improving MALDI quantification, the slopes of the calibration curves inversely correlate with peptide molecular weights; the higher molecular weight for the peptide, the lower the slope of the calibration curve. The ratio of the calibrationcurve slopes are 5.0: 2.5: 1.0 for bradykinin, melittin, and insulin, respectively. These ratios correlate well with the inverse of the analyte's molecular weight, 5.4: 2.0: 1.0. The decrease in the slope as the molecular mass increases may

Table 1. Average peak-height ratios (analyte:internal standard) and standard deviations (SDs) from the quantification of bradykinin, melittin, and bovine insulin with substance $\mathrm{P}$ as the single internal standard

\begin{tabular}{|c|c|c|c|}
\hline & Bradykinin/Sub P & Melittin/Sub P & Bovine insulin/Sub $P$ \\
\hline $\begin{array}{l}\text { Analyte/internal } \\
\text { standard ratio }\end{array}$ & $\begin{array}{c}\text { Average peak } \\
\text { height ratio } \pm S D\end{array}$ & $\begin{array}{c}\text { Average peak } \\
\text { height ratio } \pm S D\end{array}$ & $\begin{array}{l}\text { Average peak height } \\
\text { ratio } \pm S D\end{array}$ \\
\hline 0.1 & $0.17 \pm 0.02$ & $0.05 \pm 0.01$ & $0.020 \pm 0.004$ \\
\hline 0.2 & $0.30 \pm 0.02$ & $0.11 \pm 0.01$ & $0.06 \pm 0.01$ \\
\hline 0.5 & $0.49 \pm 0.02$ & $0.17 \pm 0.01$ & $0.14 \pm 0.01$ \\
\hline 1.0 & $1.2 \pm 0.1$ & $0.49 \pm 0.04$ & $0.24 \pm 0.02$ \\
\hline 2.0 & $2.1 \pm 0.2$ & $1.04 \pm 0.02$ & $0.45 \pm 0.02$ \\
\hline 5.0 & $5.1 \pm 0.1$ & $2.4 \pm 0.1$ & $1.14 \pm 0.05$ \\
\hline 10.0 & $9.9 \pm 0.7$ & $5.0 \pm 0.5$ & $2.1 \pm 0.2$ \\
\hline
\end{tabular}


be a combination of lower efficiency of desorption and detection (ion-to-electron conversion), resulting in weaker signals for analytes of higher molecular mass. Nevertheless, the good agreement between ratios of the inverse molecular masses and slopes of calibration plots suggests that there is an opportunity to predict a priori the slope of a calibration plot based on molecular weight when the hydrophobicity is comparable. We are planning experiments to test more extensively this possibility.

\section{Acknowledgments}

This research was supported by a grant from the National Centers of Research Resources of the NIH (grant P41RR00954).

\section{References}

1. Wasserscheid, P.; Keim, W. Ionic liquids-new "solutions" for transition metal catalysis. Angew. Chem. Int. Ed. Engl. 2000, 39, 3772-3789.

2. Armstrong, D. W.; He, L.; Liu, Y.-S. Examination of Ionic Liquids and Their Interaction with Molecules, When Used as Stationary Phases in Gas Chromatography. Anal. Chem. 1999, 71, 3873-3876.

3. Anderson, J. L.; Armstrong, D. W. High-Stability Ionic Liquids. A New Class of Stationary Phases for Gas Chromatography. Anal. Chem. 2003, 75, 4851-4858.

4. Armstrong, D. W.; Zhang, L.-K.; He, L.; Gross, M. L. Ionic Liquids as Matrixes for Matrix-Assisted Laser Desorption/Ionization Mass Spectrometry. Anal. Chem. 2001, 73, 3679-3686.

5. Anderson, J. L.; Ding, J.; Welton, T.; Armstrong, D. W. Characterizing Ionic Liquids on the Basis of Multiple Solvation Interactions. J. Am. Chem. Soc. 2002, 124, 14247-14254.

6. Carda-Broch, S.; Berthod, A.; Armstrong, D. W. Ionic Matrices for Matrix-Assisted Laser Desorption/Ionization Time-ofFlight Detection of DNA Oligomers. Rapid Commun. Mass Spectrom. 2003, 17, 553-560.

7. Mank, M.; Stahl, B.; Boehm, G. 2,5-Dihydroxybenzoic Acid Butylamine and Other Ionic Liquid Matrixes for Enhanced MALDI-MS Analysis of Biomolecules. Anal. Chem. 2004, 76, 2938-2950.

8. Li, Y. L.; Gross, M. L. Ionic-Liquid Matrices for Peptide Quantification and for Analysis of Phospholipids by MALDITOF MS. Proceedings of the 52nd Conference on Mass Spectrometry and Allied Topics; Nashville, TN, May, 2004.

9. Sze, E. T. P.; Dominic Chan, T. W.; Wang, G. Formulation of Matrix Solutions for Use in Matrix-Assisted Laser Desorption/Ionization of Biomolecules. J. Am. Soc. Mass Spectrom. 1998, 9, 166-174.

10. Bogan, M. J.; Agnes, G. R. Wall-less Sample Preparation of $\mu \mathrm{m}$-sized Sample Spots for Femtomole Detection Limits of Proteins from Liquid Based UV-MALDI Matrices. J. Am. Soc. Mass Spectrom. 2004, 15, 486-495.

11. Owens, K. G. Analytical Currents: Making MALDI Reproducibly Quantitative. Anal. Chem. 1998, 70, 17A-18A.

12. Garcia, B. A.; Heaney, P. J.; Tang, K. Improvement of the MALDI-TOF Analysis of DNA with Thin-Layer Matrix Preparation. Anal. Chem. 2002, 74, 2083-2091.

13. Zhang, L. K.; Gross, M. L. Matrix-Assisted Laser Desorption/ Ionization Mass Spectrometry Methods for Oligodeoxynucleotides: Improvements in Matrix, Detection Limits, Quantification, and Sequencing. J. Am. Soc. Mass Spectrom. 2000, 11, $854-865$.

14. Fan, X.; Beavis, R. C. A Method to Increase Contaminant Tolerance in Protein Matrix-Assisted Laser Desorption/Ion- ization by the Fabrication of Thin Protein-Doped Polycrystalline Films. Rapid Commun. Mass Spectrom. 1994, 8, 199-204.

15. Amado, F. M. L.; Domingues, P.; Santana-Marques, M. G.; Ferrer-Correia, A. J.; Tomer, K. B. Discrimination Effects and Sensitivity Variations in Matrix-Assisted Laser Desorption/ Ionization. Rapid Commun. Mass Spectrom. 1997, 11, 1347-1352.

16. Onnerfjord, P.; Ekstrom, S.; Bergquist, J.; Nilsson, J.; Laurell, T.; Marko-Varga, G. Homogeneous Sample Preparation for Automated High Throughput Analysis with Matrix-Assisted Laser Desorption/Ionization Time-of-Flight Mass Spectrometry. Rapid Commun. Mass Spectrom. 1999, 13, 315-322.

17. Dai, Y.; Whittal, R. M.; Li, L. Two-Layer Sample Preparation: A Method for MALDI-MS Analysis of Complex Peptide and Protein Mixtures. Anal. Chem. 1999, 71, 1087-1091.

18. Koomen, J. M.; Russell, D. H. Ultraviolet/Matrix-Assisted Laser Desorption/Ionization Mass Spectrometric Characterization of 2,5-Dihydroxybenzoic Acid-Induced Reductive Hydrogenation of Oligonucleotides on Cytosine Residues. J. Mass Spectrom. 2000, 35, 1025-1034.

19. Vorm, O.; Roepstorff, P.; Mann, M. Improved Resolution and Very High Sensitivity in MALDI TOF of Matrix Surfaces Made by Fast Evaporation. Anal. Chem. 1994, 66, 3281-3287.

20. Hensel, R. R.; King, R. C.; Owens, K. G. Electrospray Sample Preparation for Improved Quantitation in Matrix-Assisted Laser Desorption/Ionization Time-of-Flight Mass Spectrometry. Rapid Commun. Mass Spectrom. 1997, 11, 1785-1793.

21. Tanaka, K.; Waki, H.; Ido, Y.; Akita, S.; Yoshida, Y.; Yohida, T. Protein and Polymer Analyses up to $m / z$ 100,000 by Laser Ionization Time-of-Flight Mass Spectrometry. Rapid Commun. Mass Spectrom. 1988, 2, 151-153.

22. Sunner, J.; Dratz, E.; Chen, Y.-C. Graphite Surface-Assisted Laser Desorption/Ionization Time-of-Flight Mass Spectrometry of Peptides and Proteins from Liquid Solutions. Anal. Chem. 1995, 67, 4335-4342.

23. Zabet-Moghaddam, M.; Heinzle, E.; Tholey, A. Qualitative and Quantitative Analysis of Low Molecular Weight Compounds by Ultraviolet Matrix-Assisted Laser Desorption/ Ionization Mass Spectrometry Using Ionic Liquid Matrices. Rapid Commun. Mass Spectrom. 2004, 18, 141-148.

24. Wilkinson, W. R.; Gusev, A. I.; Proctor, A.; Houalla, M.; Hercules, D. M. Selection of Internal Standards for Quantitative Analysis by Matrix-Assisted Laser Desorption-Ionization (MALDI) Time-of-Flight Mass Spectrometry. Fresenius J. Anal. Chem. 1997, 357, 241-248.

25. Gusev, A. I.; Wilkinson, W. R.; Proctor, A.; Hercules, D. M. Direct Quantitative Analysis of Peptides Using Matrix Assisted Laser Desorption Ionization. Fresenius J. Anal. Chem. 1996, 354, 455-463.

26. Tang, K.; Allman, S. L.; Jones, R. B.; Chen, C. H. Quantitative Analysis of Biopolymers by Matrix-Assisted Laser Desorption. Anal. Chem. 1993, 65, 2164-2166.

27. Nelson, R. W.; McLean, M. A.; Hutchens, T. W. Quantitative Determination of Proteins by Matrix-Assisted Laser Desorption/Ionization Time-of-Flight Mass Spectrometry. Anal. Chem. 1994, 66, 1408-1415.

28. Bruenner, B. A.; Yip, T.-T.; Hutchens, T. W. Quantitative Analysis of Oligonucleotides by Matrix-Assisted Laser Desorption/Ionization Mass Spectrometry. Rapid Commun. Mass Spectrom. 1996, 10, 1797-1801.

29. Gusev, A. I.; Wilkinson, W. R.; Proctor, A.; Hercules, D. M. Quantitative Analysis of Peptides by Matrix-Assisted Laser Desorption/Ionization Time-of-Flight Mass Spectrometry. Appl. Spectrosc. 1993, 47, 1091-1092.

30. Bull, H. B.; Breese, K. Surface Tension of Amino Acid Solutions. Hydrophobicity Scale of the Amino Acid Residues. Arch. Biochem. Biophys. 1974, 161, 665-670. 